\title{
Forming an early 0-type star through gas accretion?
}

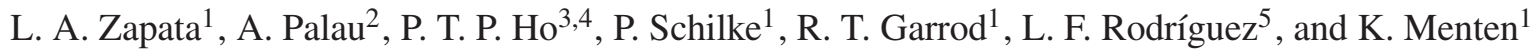 \\ 1 Max-Planck-Institut für Radioastronomie, Auf dem Hügel 69, 53121 Bonn, Germany \\ e-mail: lzapata@mpifr-bonn.mpg.de \\ 2 Laboratorio de Astrofísica Espacial y Física Fundamental, Apartado 7828691 Villanueva de la Cañada, Madrid, Spain \\ 3 Harvard-Smithsonian Center for Astrophysics, 60 Garden Street, Cambridge, MA 02138, USA \\ 4 Academia Sinica Institute of Astronomy and Astrophysics, Taipei, Taiwan \\ 5 CRyA, Universidad Nacional Autónoma de México, Apdo. Postal 3-72 (Xangari), 58089 Morelia, Michoacán, México
}

Received 12 October 2007 / Accepted 29 November 2007

\section{ABSTRACT}

\begin{abstract}
We present high angular resolution $\left(\sim 3^{\prime \prime}\right)$ and sensitive $1.3 \mathrm{~mm}$ continuum, cyanogen $(\mathrm{CN})$ and vinyl cyanide $\left(\mathrm{C}_{2} \mathrm{H}_{3} \mathrm{CN}\right)$ line observations made with the Submillimeter Array (SMA) toward one of most highly obscured objects of the W51 IRS2 region, W51 North. We find that the $\mathrm{CN}$ line exhibits a pronounced inverse P-Cygni profile indicating that the molecular gas is falling into this object with a mass accretion rate between 4 and $7 \times 10^{-2} M_{\odot} \mathrm{yr}^{-1}$. The $\mathrm{C}_{2} \mathrm{H}_{3} \mathrm{CN}$ traces an east-west rotating molecular envelope that surrounds either a single obscured (proto)star with a kinematic mass of $40 M_{\odot}$ or a small central cluster of B-type stars and that is associated with a compact high velocity bipolar outflow traced by $\mathrm{H}_{2} \mathrm{O}$ masers and $\mathrm{SiO}$ molecular emission. We thus confirm that the W51 North region is part of the growing list of young massive star forming regions that have been associated with infalling motion and with high mass accretion rates $\left(\sim 10^{-2}-10^{-4} M_{\odot} \mathrm{yr}^{-1}\right)$, strengthening the evidence that massive stars can form with very high accretion rates sufficient to quench the formation of a UCHII region.
\end{abstract}

Key words. molecular data - techniques: interferometric - stars: formation - ISM: jets and outflows - ISM: molecules radio lines: ISM

\section{Introduction}

One of the main questions related to star formation is whether massive stars $\left(>10 M_{\odot}\right)$ are formed through gas accretion via a circumstellar disk/torus or whether other mechanims play a role. It was believed that the powerful radiation fields and stellar winds produced at the beginning of nuclear burning will increasingly inhibit further accretion of material, thereby limiting the maximum stellar mass to about $10 M_{\odot}$ (Kahn 1974; Larson \& Starrfield 1971; Yorke \& Kruegel 1977). Several theoretical models have since then been proposed to solve this puzzle: the formation of massive stars through dense disks with jets/outflows (Nakano 1989; Jijina \& Adams 1996), through merging of smaller stars (Bonnell et al. 1998), through turbulent accretion (McKee \& Tan 2003), through competitive accretion (Bonnell et al. 2001), and through ionized accretion flows (Keto \& Wood 2006). However, due to the lack of good observational evidence, these alternatives have remained controversial (Zinnecker \& Yorke 2007).

With a total bolometric luminosity of about $3 \times 10^{6} L_{\odot}$ the W51-IRS2 region is one of the most luminous massive star forming regions in our Galaxy (Erickson \& Tokunaga 1980). It is located 6-7 kpc away in the Sagittarius spiral arm (Genzel et al. 1981; Imai et al. 2002). We adopt here a distance to the W51 region of $7 \mathrm{kpc}$. The W51 IRS2 region comprises a complex group of highly obscured young objects (with no mid-infrared counterparts, see Kraemer et al. 2001; Okamoto et al. 2001) called "W51 North" and "W51d2", and a cluster of massive strong infrared ZAMS stars (Kraemer et al. 2001; Okamoto et al. 2001; Lacy et al. 2007) with the most prominent member being the source "IRS2d" associated with an extended edge-brightened cometary HII region called "W51d" (Gaume et al. 1993; Lacy et al. 2007).

The W51 North object shows strong thermal dust emission at (sub)millimeter wavelengths, molecular emission from a large $\left(\sim 4 \times 10^{4} \mathrm{AU}\right)$ hot core at an excitation temperature of 100-200 K (Zhang et al. 1998), and very faint centimeter freefree emission (Gaume et al. 1993; Zhang et al. 1998; Eisner et al. 2002), suggesting that it is forming an extremely young massive star. This object, in addition, contains a group of strong $\mathrm{H}_{2} \mathrm{O}, \mathrm{OH}$ and $\mathrm{SiO}$ masers that are located in the center of this molecular and dusty structure and that is called "The Dominant Center" (Schneps et al. 1981; Gaume \& Mutel 1987; Hasegawa et al. 1986; Ukita et al. 1987; Morita et al. 1992). Observations of the proper motions of the $\mathrm{H}_{2} \mathrm{O}$ masers (which trace shocks in dustladen gas close to the exciting protostars, Elitzur 1992) revealed the presence of a compact ( $7000 \mathrm{AU})$ northwest-southeast high velocity $\left(>100 \mathrm{~km} \mathrm{~s}^{-1}\right.$ ) outflow (Schneps et al. 1981; Eisner et al. 2002; Imai et al. 2002). Moreover, high angular resolution observations showed that the $\mathrm{SiO}$ masers seem to be tracing the innermost parts of this powerful outflow (Eisner et al. 2002). This object has been identified with spectroscopic signatures of dynamical collapse using emission from $\mathrm{HCO}^{+}$(Rudolph et al. 1990), and $\mathrm{SO}_{2}$ (Sollins et al. 2004).

Here we present $1.3 \mathrm{~mm}$ continuum, cyanogen and vinyl cyanide line observations toward the W51 North region made with the SMA. We report the presence of molecular gas accretion onto a $40 M_{\odot}$ (proto)star or a small central cluster of B stars located in the center of W51 North region with an accretion rate between 4 and $7 \times 10^{-2} M_{\odot} \mathrm{yr}^{-1}$. 


\section{Observations}

The observations were obtained with the $\mathrm{SMA}^{1}$ during 2005 August 20. The SMA was in its compact configuration, which includes 21 independent baselines ranging in projected length from 16 to $50 \mathrm{~m}$. The phase reference center of the observations was $\mathrm{RA}=19^{\mathrm{h}} 23^{\mathrm{m}} 43.80^{\mathrm{s}}$, Dec $=14^{\circ} 31^{\prime} 30.0^{\prime \prime}(\mathrm{J} 2000.0)$. The frequency was centered at $217.1049 \mathrm{GHz}$ in the Lower Sideband (LSB), while the Upper Sideband (USB) was centered at $228.1049 \mathrm{GHz}$.

A close blend of the CN $N=2-1, J=5 / 2-3 / 2, F=$ $5 / 2-3 / 2$ and $N=2-1, J=5 / 2-3 / 2, F=7 / 2-5 / 2$ lines were detected in the USB. Their frequencies are 226.874166 and $226.874745 \mathrm{GHz}$, respectively. Both lines have very similar intrinsic strengths and energies above the ground state. The LSR velocity scale in this paper is given with respect to the rest frequency of the former line. The velocity difference corresponding to the frequency difference is $0.77 \mathrm{~km} \mathrm{~s}^{-1}$. When, in this paper, we refer to the $\mathrm{CN}$ line, we mean this blend. The $\mathrm{C}_{2} \mathrm{H}_{3} \mathrm{CN}$ $J_{K_{a}, K_{c}}=23_{2,22}-22_{2,21}$ line was detected in the LSB at a frequency of $217.497585 \mathrm{GHz}$.

The full bandwidth of the SMA digital correlator is $4 \mathrm{GHz}$ ( $2 \mathrm{GHz}$ in each side band). The correlator was configured with spectral windows ("chunks") of $104 \mathrm{MHz}$ each, with 128 channels distributed over each spectral window, providing a resolution of $0.8125 \mathrm{MHz}\left(1.1 \mathrm{~km} \mathrm{~s}^{-1}\right)$ per channel.

The zenith opacity measured $\left(\tau_{230 \mathrm{GHz}}\right)$ with the NRAO tipping radiometer located at the Caltech Submillimeter Observatory (close to the SMA) varied during the night between 0.12 and 0.20 , indicating good weather conditions during the observations. Phase and amplitude calibrators were the quasars 1749+096 and 1741-038, with measured flux densities and formal fitting errors of $2.08 \pm 0.05$ and $1.81 \pm$ $0.05 \mathrm{Jy}$, respectively. The uncertainty in the flux scale is estimated to be $15-20 \%$, based on the SMA monitoring of quasars. Observations of Uranus provided the absolute scale for the flux density calibration. Further technical descriptions of the SMA and its calibration schemes acan found in Ho et al. (2004).

The data were calibrated using the IDL superset MIR, originally developed for the Owens Valley Radio Observatory (Scoville et al. 1993) and adapted for the SMA ${ }^{2}$. The calibrated data were imaged and analyzed in the standard manner using the MIRIAD and AIPS packages. We used the ROBUST parameter of the INVERT task set to -2, which corresponds to uniform weighting to achieve the maximum angular resolution while sacrificing some sensitivity. The resulting image rms noise of line images was $30 \mathrm{mJy}^{\text {beam }}{ }^{-1}$ for each channel at an angular resolution of $3.4^{\prime \prime} \times 3.2^{\prime \prime}$ with a PA $=-87^{\circ}$. The data were selfcalibrated in phase and amplitude using as a model the continuum image. The final images were shifted $\sim 1^{\prime \prime}$ in right ascension in order to be consistent with the positions of the molecular cores associated with the W51 North and W51d2 better determined with the Very Large Array observations of Ho et al. (1983); Zhang \& Ho (1997). This discrepancy is mainly caused by the baseline error, the finite $\mathrm{S} / \mathrm{N}$, and the atmospheric fluctuations in our millimeter wave observations.

\footnotetext{
1 The Submillimeter Array (SMA) is a joint project between the Smithsonian Astrophysical Observatory and the Academia Sinica Institute of Astronomy and Astrophysics, and is funded by the Smithsonian Institution and the Academia Sinica.

2 The MIR-IDL cookbook by C. Qi can be found at http://cfa-www. harvard. edu/ cqi/mircook.html
}

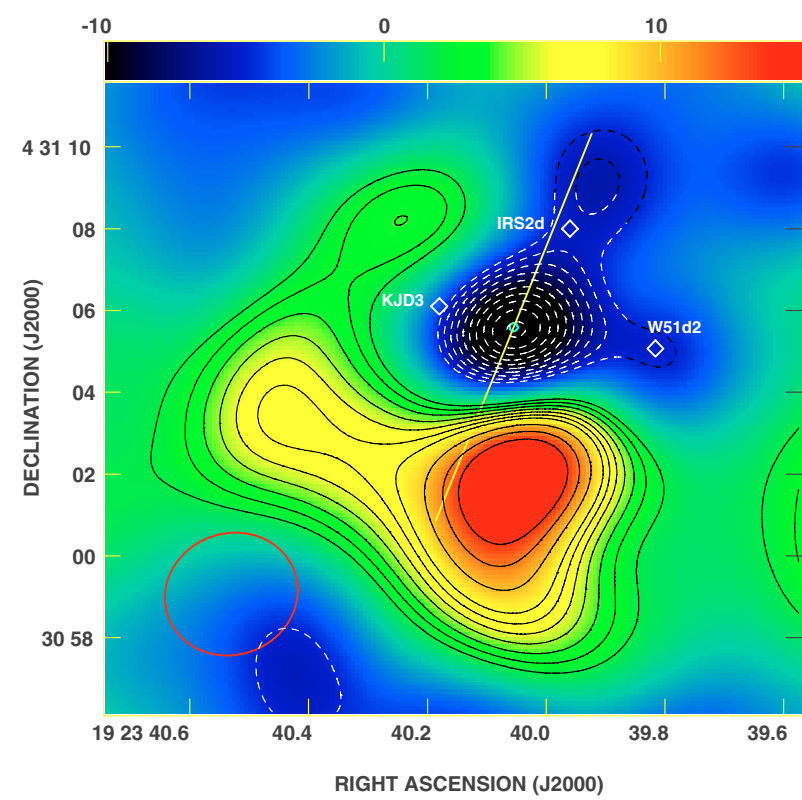

Fig. 1. CN $N=2-1, J=5 / 2-3 / 2, F=5 / 2-3 / 2$ and $N=2-1$, $J=5 / 2-3 / 2, F=7 / 2-5 / 2$ integrated line emission image of the W51 IRS2 region. The contours are $-14,-13,-12,-11,-10,-9$, $-8,-7,-6,-5,-4,4,5,6,7,8,10,11,12,13$, and 14 times the $970 \mathrm{mJy}^{-1}$ beam ${ }^{-1}$, the rms noise of the image. The integration is over a velocity range from 50 to $80 \mathrm{~km} \mathrm{~s}^{-1}$. The synthesized beam is $3.4^{\prime \prime} \times$ $3.2^{\prime \prime}$ with a PA $=-87^{\circ}$, and is shown in the bottom left corner. The scale bar indicates the molecular line emission and absorption in Jy Beam ${ }^{-1}$. The white diamonds indicate the positions of the infrared sources KJD3 and IRS2d (Kraemer et al. 2001; Okamoto et al. 2001; Lacy et al. 2007) and the radio source W51d2 (Gaume et al. 1993). The yellow line indicates the orientation of the $\mathrm{SiO}(5-4)$ high velocity bipolar molecular outflow centered on the water masers (Zapata et al., in prep.). The green circle indicates the position of the center of W51 North (Schneps et al. 1981).

\section{Results and discussion}

\subsection{The molecular and millimeter continuum emission}

In Fig. 1, we present the integrated $\mathrm{CN}$ line emission image of the W51 IRS2 region, and the positions of the $\mathrm{H}_{2} \mathrm{O}$ masers (Imai et al. 2002), the infrared sources W51 IRS2d and KJD3/OKYM1 (Kraemer et al. 2001; Okamoto et al. 2001; Lacy et al. 2007), and the radio source W51d2 (Gaume et al. 1993), all in the neighborhood of the W51 North object. We mark the position and orientation of the strong high velocity molecular bipolar outflow traced by the $\mathrm{SiO} v=0 ; J=5-4$ line found by Zapata et al. (in prep.), which is centered on the cluster of masers. In this image we can see two components of the molecular line distribution, one in unresolved absorption toward the W51 North object and the other also quite compact, but resolved in emission surrounding this region. We interpret this appearance as the signature of molecular absorption against the strong compact millimeter continuum source associated with W51 North and shown in Fig. 3.

Figure 2 shows the spectrum of the $\mathrm{CN}$ line towards the center of the W51 North object. The line shows a pronounced inverse $\mathrm{P}$ Cygni profile. The emission feature appears at the $V_{\mathrm{LSR}}=$ $55 \mathrm{~km} \mathrm{~s}^{-1}$ and the absorption at $V_{\mathrm{LSR}}=65 \mathrm{~km} \mathrm{~s}^{-1}$, which is consistent with the $\mathrm{HCO}^{+}$molecular observations of Rudolph et al. (1990). Given that the core's systemic velocity is $59 \mathrm{~km} \mathrm{~s}^{-1}$ (Zhang et al. 1998), the location of the redshifted absorption projected against the bright continuum emission of the central highly obscured object implies inward motion away from the 


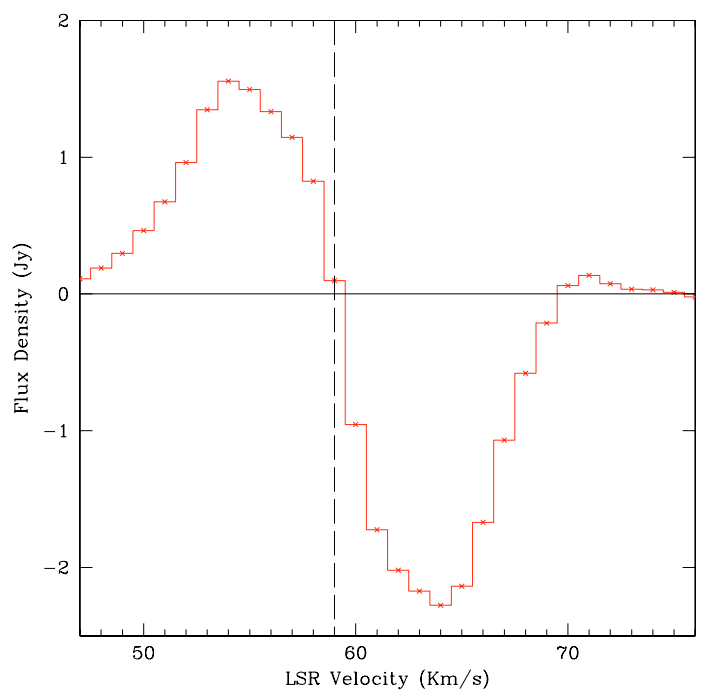

Fig. 2. Spectrum of the CN $N=2-1, J=5 / 2-3 / 2, F=5 / 2-3 / 2$ and $N=2-1, J=5 / 2-3 / 2, F=7 / 2-5 / 2$ lines towards the center of the W51 North object. The spectral velocity resolution is $1.1 \mathrm{~km} \mathrm{~s}^{-1}$. The dashed line indicates the systemic velocity $\left(V_{\mathrm{LSR}}=59 \mathrm{~km} \mathrm{~s}^{-1}\right)$.

observer. The brightness temperature of the emission from the infalling material is lower than $\sim 10 \mathrm{~K}$, the brightness temperature of the continuum emission from the core. From Fig. 6 of Rudolph et al. (1990) and Fig. 2 shown here, we estimate that the velocity of infall $\left(V_{\text {infall }}\right)$ is about $4 \mathrm{~km} \mathrm{~s}^{-1}$. With this information and taking the values of the density $\left(\rho=2 \times 10^{6} \mathrm{~cm}^{-3}\right)$, linear radius $\left(r=1.4 \times 10^{4} \mathrm{AU}\right)$ reported for the compact continuum source located in W51 North (Zhang \& Ho 1997; Zhang et al. 1998) and a radius of the hot core of $2 \times 10^{4} \mathrm{AU}$, and following Beltran et al. (2006), we calculate that the mass infall rate $(\dot{M}=$ $\left.4 \pi r^{2} \rho V_{\text {infall }}\right)$ is between 4 and $7 \times 10^{-2} M_{\odot} \mathrm{yr}^{-1}$. This value has large uncertainties, due to the uncertainty on the density and on the radius at which $V_{\text {infall }}$ is measured.

The first moment map of the $\mathrm{C}_{2} \mathrm{H}_{3} \mathrm{CN} J_{K_{a}, K_{c}}=$ $23_{2,22}-22_{2,21}$ line is shown in Fig. 3. The emission traces an unresolved east-west rotating molecular "envelope" or "core" with a total velocity shift of $1.5 \mathrm{~km} \mathrm{~s}^{-1}$ and a size of $4 \times 10^{4} \mathrm{AU}$. Moreover, the integrated emission from this molecule is well centered on the millimeter continuum source, suggesting that this specie traces high density gas close to the (proto)star (see Fig. 3). Our bandpasses contained lines from other molecules (e.g. $\mathrm{HCOOCH}_{3}$ and $\mathrm{CH}_{3} \mathrm{OH}$ ) associated with W51 North. However, they were very much contaminated by the emission from the hot molecular core associated with W51d2, not allowing us to search for similar east-west velocity gradients associated with these line molecular tracers. The W51 North source is associated with the extended hot core found by Ho et al. (1983) and Zhang et al. (1998), and it is centered on the cluster of masers as reported in other observations e.g., $\mathrm{NH}_{3}$; $\mathrm{Ho}$ et al. (1983); Eisner et al. (2002), $\mathrm{CH}_{3} \mathrm{CN}$; Zhang et al. (1998), $\mathrm{SO}_{2}$; Sollins et al. (2004). If we assume that the molecular gas is rotating as a rigid body (i.e. the dynamical mass is $M_{\mathrm{dyn}}=$ $v^{2} r \sin ^{2}(i) / G$, where $v$ is the rotation velocity, $r$ is the radius of the envelope, $i$ is the inclination angle of the envelope assumed to be $90^{\circ}$ and $G$ is the gravitational constant), we estimate a mass for the central object(s) of $40 M_{\odot}$. This central object might be associated with a single central O-type (proto)star or with a small group of B-type (proto)stars. However, as there is a strong and compact bipolar outflow in the center of the core (see Fig. 3), that seems to be dominated by one central massive star.
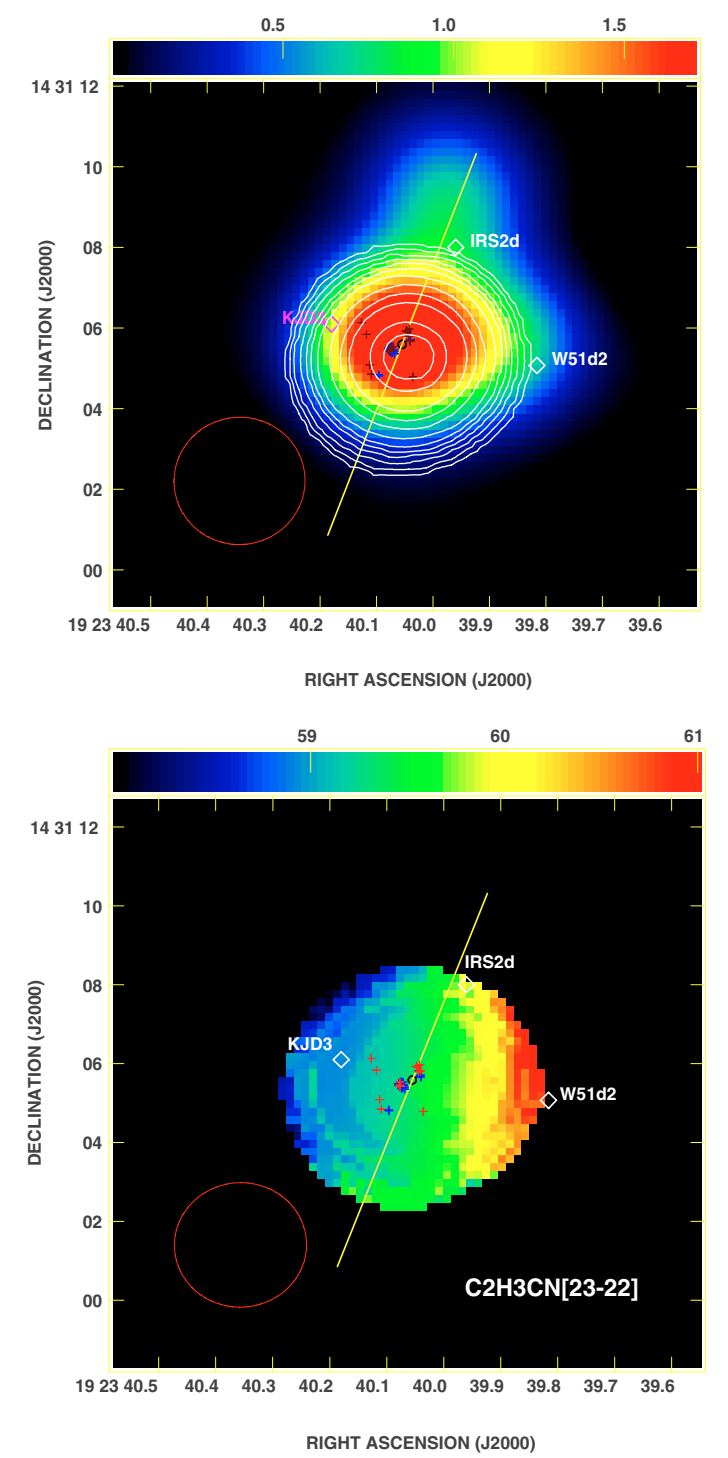

Fig. 3. Upper: SMA $1.3 \mathrm{~mm}$ continuum emission color image overlayed with the moment zero distribution of the $\mathrm{C}_{2} \mathrm{H}_{3} \mathrm{CN}$ line (pink contours) of the W51 North region. The integration is over a velocity range of 55 to $65 \mathrm{~km} \mathrm{~s}^{-1}$. The synthesized beam with a FWHM 3.4" $\times 3.2^{\prime \prime}$ and a PA of $-87^{\circ}$ is shown in the bottom left corner. The contours are -4 , $4,8,12,16,20,24,30,40,50,60,80,90,100,120,150,170$ and 200 times $40 \mathrm{mJy} \mathrm{beam}{ }^{-1} \mathrm{~km} \mathrm{~s}^{-1}$, the rms noise of the image. The scale bar indicates the continuum peak flux density in $\mathrm{Jy} \mathrm{beam}^{-1}$. Lower: first moment color image of the $\mathrm{C}_{2} \mathrm{H}_{3} \mathrm{CN}$ emission toward the W51 North region. The integration is over a velocity range of 55 to $65 \mathrm{~km} \mathrm{~s}^{-1}$. The scale bar indicates the velocity shift in $\mathrm{km} \mathrm{s}^{-1}$. The white diamonds indicate the positions of the infrared sources KJD3 and IRS2d (Kraemer et al. 2001; Okamoto et al. 2001; Lacy et al. 2007) and the radio source W51d2 (Gaume et al. 1993). The blue and red crosses indicate the position of the blue- and red-shifted strong $\mathrm{H}_{2} \mathrm{O}$ masers spots, respectively, reported by Imai et al. (2002). Note that the central cluster of masers is tracing a high velocity outflow with a northwest-southeast orientation (Imai et al. 2002; Eisner et al. 2002). The yellow line indicates the orientation of the $\mathrm{SiO}(5-4)$ high velocity bipolar outflow centered on the water masers (Zapata et al., in prep.). The black circle indicates the position of the center of W51 North (Schneps et al. 1981).

From Fig. 3 and assuming that at a wavelength of $1.3 \mathrm{~mm}$ we are observing isothermal optically thin dust emission with a dust mass opacity coefficient that varies with frequency as $\kappa \propto \nu^{\beta}$, with $\beta=1$ (the size of the source suggest that we are observing 
emission from the envelope; hence, we adopt $\beta=1$, however, this value is uncertain, see Beckwith et al. (1990) who observe how this value varies in pre-main-sequence stars), a gas-to-dust ratio of 100, which may not be the most adequate to use for protostellar sources since erosion of the circumstellar envelope by photoevaporation from near OB stars may decrease the gas-todust ratio, see Williams et al. (2005); Throop \& Bally (2005), an adopted value of $\kappa_{1.3 \mathrm{~mm}}=1.5 \mathrm{~cm}^{2} \mathrm{~g}^{-1}$ and a dust temperature value of about $100 \mathrm{~K}$ (with uncertainties of a factor of about 1.5, Zhang et al. 1998), we estimate an enclosed mass of the molecular core W51 North of $90 M_{\odot}$, very close to the $100 M_{\odot}$ estimated by Zhang \& Ho (1997). Due to the uncertainties referred to above, the values of the derived masses are accurate within a factor of 2 .

\subsection{Forming an early O-type star in W51 North?}

The combined $1.3 \mathrm{~mm}$ continuum, $\mathrm{C}_{2} \mathrm{H}_{3} \mathrm{CN}$ and $\mathrm{CN}$ data from W51 North suggest that this object is forming a massive O5-type (proto)star in its center through molecular gas accretion and with a very high accretion rate between 4 and $7 \times$ $10^{-2} M_{\odot} \mathrm{yr}^{-1}$. Moreover, the powerful compact bipolar high velocity $\left(>100 \mathrm{~km} \mathrm{~s}^{-1}\right)$ outflow traced by the $\mathrm{H}_{2} \mathrm{O}$ masers pinpoints the position of this putative central massive object.

Zapata et al. (in prep.) in addition found a compact high velocity $\mathrm{SiO}$ bipolar outflow with both unresolved lobes spatially separated by less than one arcsecond and forming a PA of $150^{\circ} \pm 30^{\circ}$. This is centered at the position of the compact $\mathrm{H}_{2} \mathrm{O}$ outflow which has a PA of $110-145^{\circ}$ (Schneps et al. 1981; Imai et al. 2002; Eisner et al. 2002). We propose that these two outflows might be manifestations of a single powerful outflow. The masers appear to trace the innermost regions of it (as observed in other outflows, e.g. IRAS 20126+4104, Moscadelli et al. 2000), while the $\mathrm{SiO}$ traces the more extended shocked molecular gas. From this point of view, the southeastern cluster of $\mathrm{H}_{2} \mathrm{O}$ masers (see Fig. 3) may trace another older ejected bow-shock. However, higher angular resolution $\mathrm{SiO}$ molecular observations are neccesary to confirm this picture. The PA of the velocity gradient across the $\mathrm{C}_{2} \mathrm{H}_{3} \mathrm{CN}$ envelope $\left(\sim 90^{\circ}\right)$ is not exactly perpendicular to the orientation of the molecular outflow $\left(\mathrm{PA} \sim 140^{\circ}\right.$ ), as might be expected. This suggests that the outflow could be precessing due the presence of a binary system or that the $\mathrm{C}_{2} \mathrm{H}_{3} \mathrm{CN}$ emission is contaminated by the outflow. This physical phenomenon of precessing outflows has been reported in other outflows: IRAS 20126+4104 (Shepherd et al. 2000), L1157 (Bachiller et al. 2001), and NGC 7538IRS1 (Kraus et al. 2006).

At present, there is a list of early massive (proto)stars that have been associated with possible infalling motion and with high mass accretion rates, e.g. W51e2: a gas mass of $200 M_{\odot}$ and an accretion rate of $10^{-3} M_{\odot} \mathrm{yr}^{-1}$ (Zhang \& Ho 1997; Ho \& Young 1996); NGC 7538-IRS9: a gas mass of 100-300 $M_{\odot}$ and an accretion rate of $10^{-3} M_{\odot} \mathrm{yr}^{-1}$ (Sandell et al. 2005); G24.78+0.08: a (proto)stellar mass of $\sim 20 M_{\odot}$ and an accretion rate between $10^{-2}$ to $10^{-4} M_{\odot} \mathrm{yr}^{-1}$ (Beltrán et al. 2006);
IRAS 16547-4247: associated with an O-type (proto)star and an accretion rate of about $10^{-2} M_{\odot} \mathrm{yr}^{-1}$ (Garay et al. 2007) and W51 North, a (proto)stellar mass of $\sim 40 M_{\odot}$ and an accretion rate between $4-7 \times 10^{-2} M_{\odot} \mathrm{yr}^{-1}$ (these results). This suggests that the very massive stars (O-type) might form starting with very high accretion rates, sufficient to quench the formation of an UCHII region. This hypothesis has been proposed during recent numerical simulations (Banerjee \& Pudritz 2007).

Acknowledgements. We thank the anonymous referee for many valuable suggestions. R.G. is grateful to the Alexander von Humboldt Foundation for a Humboldt Research Fellowship.

\section{References}

Bachiller, R., Pérez Gutiérrez, M., Kumar, M. S. N., \& Tafalla, M. 2001, A\&A, 372,899

Banerjee, R., \& Pudritz, R. E. 2007, ApJ, 660, 479

Beckwith, S. V. W., Sargent, A. I., Chini, R. S., \& Guesten, R. 1990, AJ, 99, 924 Beltrán, M. T., Cesaroni, R., Codella, C., et al. 2006, Nature, 443, 427

Bonnell, I. A., Bate, M. R., \& Zinnecker, H. 1998, MNRAS, 298, 93

Bonnell, I. A., Bate, M. R., Clarke, C. J., \& Pringle, J. E. 2001, MNRAS, 323, 785

Eisner, J. A., Greenhill, L. J., Herrnstein, J. R., Moran, J. M., \& Menten, K. M. 2002, ApJ, 569, 334

Erickson, E. F., \& Tokunaga, A. T. 1980, ApJ, 238, 596

Garay, G., Mardones, D., Bronfman, L., et al. 2007, A\&A, 463, 217

Gaume, R. A., \& Mutel, R. L. 1987, ApJS, 65, 193

Gaume, R. A., Johnston, K. J., \& Wilson, T. L. 1993, ApJ, 417, 645

Genzel, R., Downes, D., Schneps, M. H., et al. 1981, ApJ, 247, 1039

Hasegawa, T., Morita, K., Okumura, S., et al. 1986, in Masers, Molecules, and

Mass Outflows in Star Formation Regions, 275

Ho, P. T. P., \& Young, L. M. 1996, ApJ, 472, 742

Ho, P. T. P., Das, A., \& Genzel, R. 1983, ApJ, 266, 596

Ho, P. T. P., Moran, J. M., \& Lo, K. Y. 2004, ApJ, 616, L1

Imai, H., Watanabe, T., Omodaka, T., et al. 2002, PASJ, 54, 741

Jijina, J., \& Adams, F. C. 1996, ApJ, 462, 874

Kahn, F. D. 1974, A\&A, 37, 149

Keto, E., \& Wood, K. 2006, ApJ, 637, 850

Kraemer, K. E., Jackson, J. M., Deutsch, L. K., et al. 2001, ApJ, 561, 282

Kraus, S., Balega, Y., Elitzur, M., et al. 2006, A\&A, 455, 521

Lacy, J. H., Jaffe, D. T., Zhu, Q., et al. 2007, ApJ, 658, L45

Larson, R. B., \& Starrfield, S. 1971, A\&A, 13, 190

McKee, C. F., \& Tan, J. C. 2003, ApJ, 585, 850

Morita, K.-I., Hasegawa, T., Ukita, N., Okumura, S. K., \& Ishiguro, M. 1992, PASJ, 44, 373

Nakano, T. 1989, ApJ, 345, 464

Okamoto, Y. K., Kataza, H., Yamashita, T., Miyata, T., \& Onaka, T. 2001, ApJ, 553,254

Rudolph, A., Welch, W. J., Palmer, P., \& Dubrulle, B. 1990, ApJ, 363, 528

Sandell, G., Goss, W. M., \& Wright, M. 2005, ApJ, 621, 839

Schneps, M. H., Moran, J. M., Genzel, R., et al. 1981, ApJ, 249, 124

Scoville, N. Z., Carlstrom, J. E., Chandler, C. J., et al. 1993, PASP, 105, 1482

Shepherd, D. S., Yu, K. C., Bally, J., \& Testi, L. 2000, ApJ, 535, 833

Sollins, P. K., Zhang, Q., \& Ho, P. T. P. 2004, ApJ, 606, 943

Throop, H. B., \& Bally, J. 2005, ApJ, 623, L149

Ukita, N., Hasegawa, T., Kaifu, N., et al. 1987, in Star Forming Regions, ed. M. Peimbert \& J. Jugaku, IAU Symp., 115, 178

Williams, S. J., Fuller, G. A., \& Sridharan, T. K. 2005, A\&A, 434, 257

Yorke, H. W., \& Kruegel, E. 1977, A\&A, 54, 183

Zhang, Q., \& Ho, P. T. P. 1997, ApJ, 488, 241

Zhang, Q., Ho, P. T. P., \& Ohashi, N. 1998, ApJ, 494, 636

Zinnecker, H., \& Yorke, H. W. 2007, ArXiv e-prints, 707 